\title{
Apert's Syndrome: rare variant of a common anomaly (Craniosynostosis)
}

\author{
Zalak Shah', Dhanya Soodhana ${ }^{2}$
}

${ }^{1}$ Dr Zalak Shah- Senior Resident, Department of Pediatrics, P.D.U Medical College, Rajkot, Gujarat, ${ }^{2}$ Dr Dhanya Soodhana- Resident, Department of Pediatrics, P.D.U Medical College, Rajkot, Gujarat.

Address for Correspondence: Zalak Shah- Address 'shivranjani',7- Niranjani Society, near Rameshwar Chowk, Raiya road, Rajkot, E-mail id: drzmupadhyay@yahoo.co.in, Dhanya Soodhana- Email id: dhanyasoodhana@gmail.com

\begin{abstract}
Apert's syndrome (Acrocephalosyndactyly) is a rare congenital condition characterized by primary craniosynostosis, mid face malformations and symmetrical syndactyly of the hand and feet. Untreated craniosynostosis leads to inhibition of brain growth and an increase in intracranial and intraorbital pressure. We present here a case of neonate with Apert's syndrome. The typical features as described makes it easy to diagnose Apert's syndrome. Radiology plays an important role in the evaluation and management of these patients. Despite of tremendous advances which have been made in the prevention and treatment of developmental anomalies, they still remain a significant cause of morbidity worldwide. Because of the multiple alterations in patients with Apert's syndrome, a multidisciplinary approach is essential for a successful planning and treatment.
\end{abstract}

Keywords: Apert's syndrome, Craniosynostosis, syndactyly

\section{Introduction}

Craniosynostosis refers to the premature fusion of one or more cranial sutures. Virchow noted that there is a cessation of growth in a direction perpendicular to that of the affected suture while growth proceeds in a parallel direction. There are also distinct craniofacial synostosis syndromes that share common features such as suture synostosis, midface hypoplasia and facial and limb abnormalities [1]. Craniosynostosis is classified as primary or secondary.

Primary craniosynostosis refers to closure of 1 or more sutures owing to abnormalities of skull development, whereas secondary craniosynostosis results from failure of growth and expansion. The incidence of primary craniosynostosis approximates 1 in 2000 live births. The cause is unknown in majority, however, genetic syndromes account for $10-20 \%$ [2]. Apert's syndrome is one such syndrome which is characterized by craniosynostosis, midface hypoplasia and symmetric syndactyly of both hands and feet. The birth prevalence is 1 in every 65000 live births [3]. We report one such case.

Manuscript received: $28^{\text {th }}$ March 2016 Reviewed: $09^{\text {th }}$ April 2016

Author Corrected; $18^{\text {th }}$ April 2016

Accepted for Publication: $30^{\text {th }}$ April 2016

\section{Case Report}

A two day old boy was received with the complain of symmetric syndactyly of both hands and feet, abnormal shape of the head and noisy breathing. The child third by order and surprisingly is the first to be affected, thus being a sporadic condition (autosomal dominant type is common).

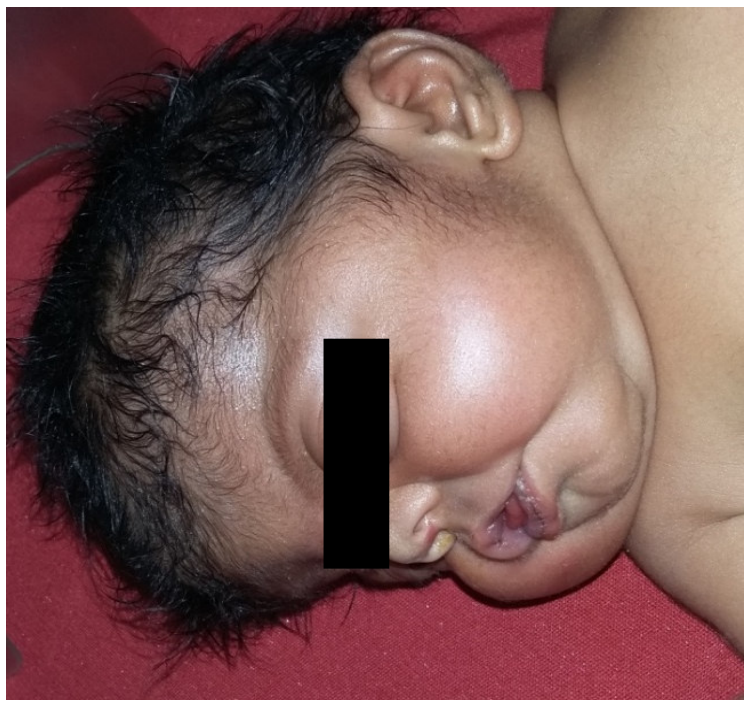

Figure 1: Apert's syndrome-facial features 


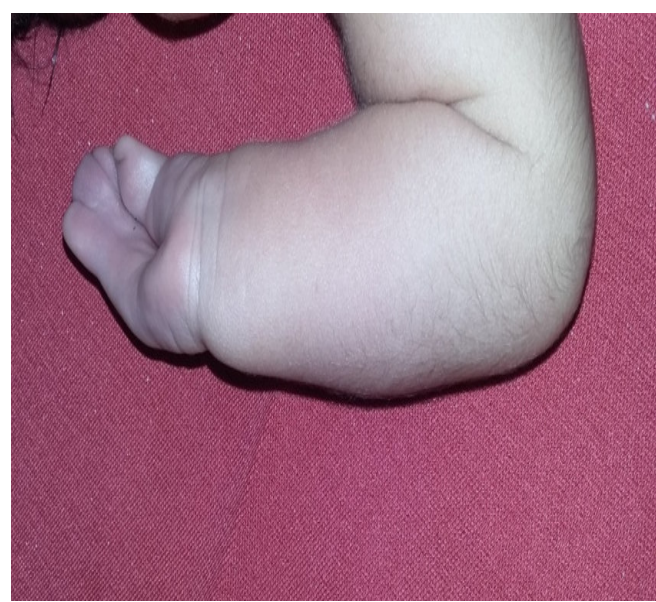

Figure 2: Mitten hands

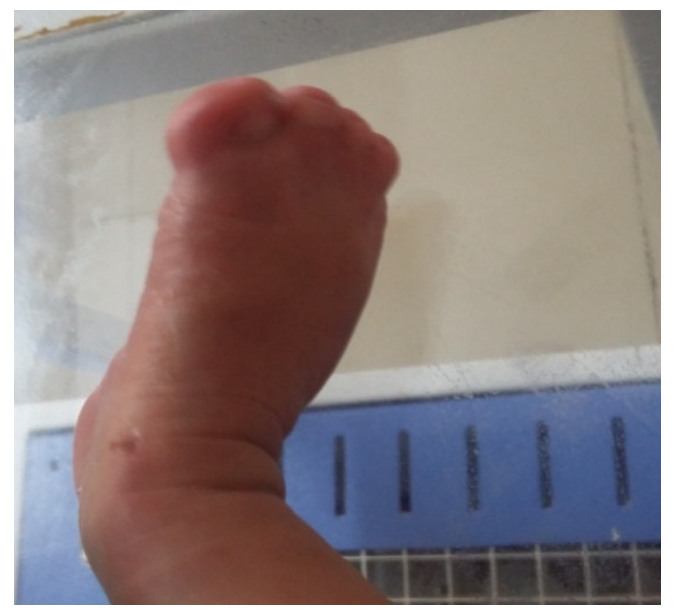

Figure 3: Socks like feet

Examination of the patient revealed the findings of abnormal contour of the head (brachycephaly), midface hypoplasia, proptosis, high arched palate, symmetric syndactyly of all the digits of the hands (mitten variety) and all the toes of the feet(socks like feet) (as seen above in the images).A provisional diagnosis of Apert's Syndrome was established and radiological investigations were performed.

Radiographs of both hands and feet showed soft tissue syndactyly of all five fingers and toes. Skull radiographs revealed fused coronal sutures, turribrachycephalic skull contour, elongated flat forehead with bitemporal widening and hypertelorism. USG abdomen and KUB was normal. ECHO was normal. Baby had noisy breathing, but no major anomaly was noticed, and was kept under observation. The baby developed jaundice on the third day and phototherapy was given for three days. Patient was referred to plastic surgeon for correction and immobilization of thumb as early as possible to develop the grasp and facial features. Patient was later discharged and was explained prognosis for this anomaly.

\section{Discussion}

Apert's syndrome makes up approximately $4 \%$ of all cases of craniosynostosis. The molecular basis of this syndrome appears remarkably specific: two adjacent amino acid substitutions (either S252W or P253R) occurring in the linking region between the second and third immunoglobulin domains of the fibroblast growth factor FGFR 2 gene [5]. Individuals with P253R mutation respond better to craniofacial surgery but have more pronounced severity of syndactyly than those with the S252W mutation. Different mutations of the same gene cause Crouzon syndrome as well as Pfeiffer syndrome [3]

The typical features as described makes it easy to diagnose Apert's syndrome. Radiology has an important role to play in the evaluation, management and follow up of these patients. Plain radiographs are sufficient for diagnosis but CT has added a new dimension to the evaluation of these disorders.

In Apert's syndrome, or acrocephalosyndactly, the cranial vault deformity is variable but most often presents as a short anteroposterior dimension with craniosynostosis involving the coronal sutures resulting in a turribrachycephalic skull. The typical craniofacial appearance includes a flat, elongated forehead with bitemporal widening and occipital flattening. There is also midface hypoplasia accompanied by orbital proptosis, downslanting palpebral fissures and hypertelorism. High arched palate, clefts of the secondary palate and crowding of the dental arch can also be seen. The nose is down turned at the tip, the bridge is depressed and the septum deviated.

Plain skull radiographs including anteroposterior (AP), lateral and Towne's projections are usually done. Now, three-dimensional CT scans have added a further dimension in planning surgery of these patients and for objective assessment of operative outcome. The dataset also enables construction of three-dimensional models on which complex surgical corrective procedures can be tested [5].

Other central nervous system abnormalities include malformations of the corpus callosum, the limbic structures, or both, megalencephaly, gyral abnormalities, pyramidal tract abnormalities, hypoplasia 
of cerebral white matter and heterotopic gray matter [6]. CT can help in the detection of such abnormalities. There is also an increased incidence of delayed mental development in these children, but many of them develop normal intelligence [6].

Cervical spine involvement in the form of variable degrees of fusion has been described. Patient may have associated occasional abnormalities- short humerus, synostosis of radius and humerus, limitation of joint mobility, genu valga, gastrointestinal anomalies in $1.5 \%$, respiratory anomalies in $1.5 \%$, cardiac defects in $10 \%$ including pulmonary stenosis, VSD, overriding of aorta, endocardial cushion defects, genitourinary anomalies in $10 \%$ [3].

The upper extremities are shortened. The usual hand abnormality in Apert's syndrome consists of a bony fusion of the second, third and fourth fingers, with a single common nail. Involvement of the first or fifth digits in this bony mass is variable. There can be a similar deformity involving the foot (mitten hand and sock foot)[7].

Early surgery is indicated when there is evidence of increased intracranial pressure. However early surgery does not prevent mental retardation, which is most likely related to malformations of central nervous system. There should be vigorous early management of the syndrome. When thumb is immobilized, early surgery to allow for a pincer grasp is indicated, with later attempts at further improvement of hand function. Newer techniques allow for vastly improved facial cosmetic reconstruction [3].

Despite of tremendous advances which have been made in the prevention and treatment of developmental anomalies, they still remain a significant cause of morbidity worldwide, which could be prevented by proper antenatal screening and counseling. Because of the multiple alterations in patients with Apert's syndrome, a multidisciplinary approach, including dentists and neurosurgeons, plastic surgeons, ophthalmologists and geneticists, is essential for a successful planning and treatment.

Source of Support: Nil, Conflict of Interest: None Permission of IRB: Yes

\section{References}

1. Upadhyaya V, Upadhyaya D N, Sarkar S. Apert's syndrome - A case report. Indian J Radiol Imaging 2005; 15:477-80

2. Stephen LK, Michael VJ. Craniosynostosis.In:Nelson textbook of Pediatrics-first south Asia edition 2015.Elsevier Inc; p2817-2819

3. Jones KL, Jones MC,Campo M.Smith's Recognizable Patterns of Human Malformation. 2013:7:474-75

4. Lajeunie E, Cameron R, El Ghouzzi V, de Parseval $\mathrm{N}$, Journeau $\mathrm{P}$, Gonzales $\mathrm{M}$, Delezoide $\mathrm{AL}$, Bonaventure J, Le Merrer M, Renier D. Clinical variability in patients with Apert's syndrome. J Neurosurg. 1999 Mar;90(3):443-7.

5. Apert E. De lacricephalosyndactylie. Bull Soc Med Hop Paris 1906; 23: 1310-1330.

6. Aviv RI, Rodger E, Hall CM. Craniosynostosis. Clin Radiol. 2002 Feb;57(2):93-102.

7. Cohen MM Jr, Kreiborg S. Skeletal abnormalities in the Apert syndrome. Am J Med Genet. 1993 Oct $1 ; 47(5): 624-32$.

\section{How to cite this article?}

Zalak Shah, Dhanya Soodhana. Apert's Syndrome: rare variant of a common anomaly (Craniosynostosis): Int J Pediatr Res 2016;3(4):263-265.doi:10.17511/ijpr.2016.i04.10. 